\title{
EPOS - Using Robotics for RvD Simulation of On-Orbit Servicing Missions
}

\author{
T. Boge ${ }^{1}$, T. Wimmer ${ }^{2}$, O. Ma ${ }^{3}$, T. Tzschichholz ${ }^{4}$ \\ German Aerospace center (DLR), Wessling, 81241, Germany
}

\begin{abstract}
Increasing complexity and costs of satellite missions promote the idea of extending the operational lifetime or improving functionalities/performance of a satellite in orbit instead of simply replacing it by a new one. Further, satellites in orbit can severely be affected by aging or degradation of their components and systems as well as by consumption of available resources. These problems may be solved by satellite on-orbit servicing (OOS) missions. One of the critical issues of such a mission is to ensure a safe and reliable Rendezvous and Docking (RvD) operation performed autonomously in space. Due to the high risk associated with an RvD operation, it must be carefully analyzed, simulated and verified in detail before the real space mission can be launched. This paper describes a ground-based hardware-inthe-loop RvD simulation facility. Designed and built on 2-decade experience of RvD experiment and testing, this unique, high-fidelity simulation facility is capable of physically simulating the final approach within 25-meter range and the docking/capture process of an on-orbital servicing mission.
\end{abstract}

$\begin{array}{ll}\text { NEOS } & =\text { German OOS technology demonstration mission } \\ D L R & =\text { German Aerospace Center } \\ E P O S & =\text { European Proximity Operation Simulator } \\ F O V & =\text { Field of View } \\ G S O C & =\text { German Space Operations Center } \\ K U K A & =\text { German robot manufacturer } \\ L E O & =\text { Low Earth Orbit } \\ O E & =\text { DARPA's Orbital Express mission } \\ O L E V & =\text { Orbital Lifetime Extension Vehicle } \\ O O S & =\text { On-Orbit Servicing } \\ R v D & =\text { Rendezvous and Docking }\end{array}$

\section{Introduction}

Meanwhile, OOS has become part of the space programs of the US, Japan, Canada and Germany. A milestone was set with the successful completion of DARPA's Orbital Express ${ }^{16}(\mathrm{OE})$ mission in 2007. The goal of OE was to demonstrate the ability to autonomously perform Rendezvous \& Docking (RvD) operations including maintenance activities like refueling. In contrast to the goals of OE, the focus of DLR is to capture non-cooperative and/or not specially prepared client spacecraft. "Non-cooperative" is understood as there is no cooperation with respect to attitude and orbit control of the client, e.g. when the client is out of operation. "Not specially prepared" means that the client satellite does not have a special docking port or retro reflectors used for vision based navigation.

\footnotetext{
${ }^{1}$ Spaceflight Technology Section, DLR, GSOC, toralf.boge@dlr.de

${ }^{2}$ Spaceflight Technology Section, DLR, GSOC, tilman.wimmer@dlr.de

${ }^{3}$ Guest Scientist at GSOC, DLR, GSOC, ou.ma@dlr.de

${ }^{4}$ Spaceflight Technology Section, DLR, GSOC, tristan.tzschichholz@dlr.de
} 


\section{A. New Challenges for RvD in Space}

Rendezvous and Docking is state of the art for manned spaceflight missions today. In addition fo the new OOS applications new technological requirements can be found for:

- Rendezvous phase

Typically the target satellites have not been built for rendezvous and docking tasks. Therefore the rendezvous sensors and systems have to cope with completely uncooperative targets.

- Docking phase

The robotic based mechanisms have to ensure a safe and reliable gripping or docking at a target without any foreseen docking mechanisms.

- Degree of Autonomy

For missions without continuous contact to ground (typically LEO missions)), the on-board autonomy plays an important role.

The new technological challenges are mainly related to OOS missions because the RvD process has to cope with uncooperative targets. Therefore one of the challenges of such OOS missions is to ensure a safe and reliable Rendezvous and Docking (RvD) process. Especially this phase has to be analyzed, simulated and verified in detail. Classical approaches e.g. numerical simulations deliver only limited results. Therefore simulation procedures, tests and the appropriate testing facilities have to be defined allowing the entire RvD process (including the flight HW of GNC components and systems) can be simulated and tested under utmost realistic conditions of the space environment.

\section{B. Reference OOS-Missions}

Recently, several satellite projects have focused on providing on-orbit servicing (OOS) capabilities in the near future. The scenarios involve an on-orbit servicing spacecraft approaching and docking to a client satellite. The paper is based on the following two reference mission scenarios.

\section{OLEV}

OLEV is a purely commercial project managed by a European consortium including a strong DLR participation. The business case of OLEV is to build an orbital servicer which is able to dock on high value, geostationary communication satellites and to take over attitude and orbit control in order to extend the clients lifetime after its fuel has been depleted (see fig. 1) Beside life extension OLEV can be used for fleet management purposes like relocation to other GEO positions or disposal to graveyard orbit.

The core element of OLEV is the capturing tool (patented by DLR) which enables OLEV to dock on the apogee engine of the majority of the existing geostationary communication satellites. The capturing tool is designed to dock / undock for several times. The OLEV platform is equipped with six "Hall Effect Thrusters" (HET): Two of them are used for the transfer from GTO to GEO, the other

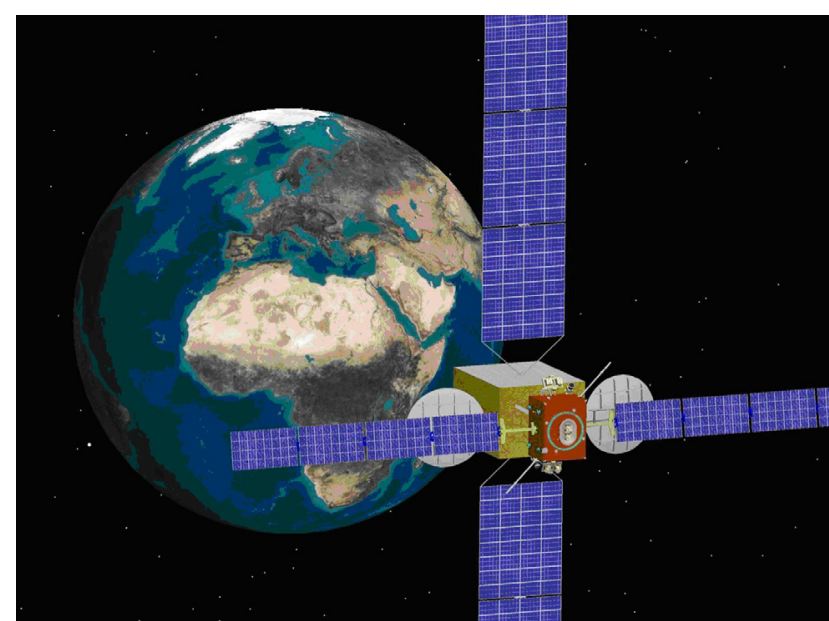

Figure 1. SMART-OLEV docked at a Geostationary satellite

four $(2 \times 2)$ are dedicated for the station keeping. The electric propulsion system enables OLEV to perform station keeping of the mated configuration for approximately twelve years depending on the client mass.

The navigation concept of OLEV is to use ranging for absolute navigation and to hand over to relative navigation at the distance of a two kilometer. For relative navigation a set of six rendezvous cameras (far, mid and close range, redundant) is used.

The OLEV project has finished a delta phase B study; the present focus lies on financial engineering.

\section{2. $D E O S$}

The primary goals of the technology demonstrator DEOS are

(1) to capture a tumbling non-cooperative client satellite with a servicer spacecraft and

(2) to de-orbit the coupled configuration within a pre-defined orbit corridor at end of mission. 
Secondary goals are to perform several rendezvous, capture and docking scenarios as well as orbit maneuvers with the mated configuration. Therefore the servicer is equipped with an active AOCS and both a manipulator and a docking port (see Figure 2).

Since the initial experiment conditions like tumbling rate of the client have to be set several times, the client is provided with an active ACS. For DEOS the expression "non-cooperative client" has to be understood in a sense that the client shall simulate a non-cooperative client. This means:

- The client has no markers or retro reflectors for navigation purposes

- The AOCS is switched of during docking or grasping process

The sensor system used for the nominal approach navigation is a vision based system using mono/stereo cameras and LIDAR.

Similar to Orbital Express the mission philosophy is to subsequently "crawl, walk and run": Both spacecraft, client and servicer, will be injected together in an initial low earth orbit (LEO). Starting with the mated configuration the complexity of the experiments is stepwise increased over mission period. One of the challenges operating DEOS is the continuity of a communication link from ground to LEO. Therefore the DEOS servicer will be equipped with a Ka-Band link to Geo-Relay satellite as an option to direct space to ground communication. The DEOS project is presently entering a phase B study financed by the German Space Agency.

\section{EPOS 2.0}

\section{A. Experiences}

DLR has more than two decades of experience in the field of simulating RvD maneuvers. The previous EPOS facility was a test bed jointly developed by ESA and DLR for the simulation of spacecraft maneuvers notably over the last few critical meters of the rendezvous phase (prior to physical docking). As shown in Figure 3, the facility consists of a large mobile platform used to hold the RvD hardware interface. The platform can provide 6-DOF translational and rotational motion to the RvD interface. The last intensive utilization of the facility was the test and verification of the ATV RvD sensors and systems which are used for the approach to ISS. It was also used for testing RvD sensors of the Japanese HTV.
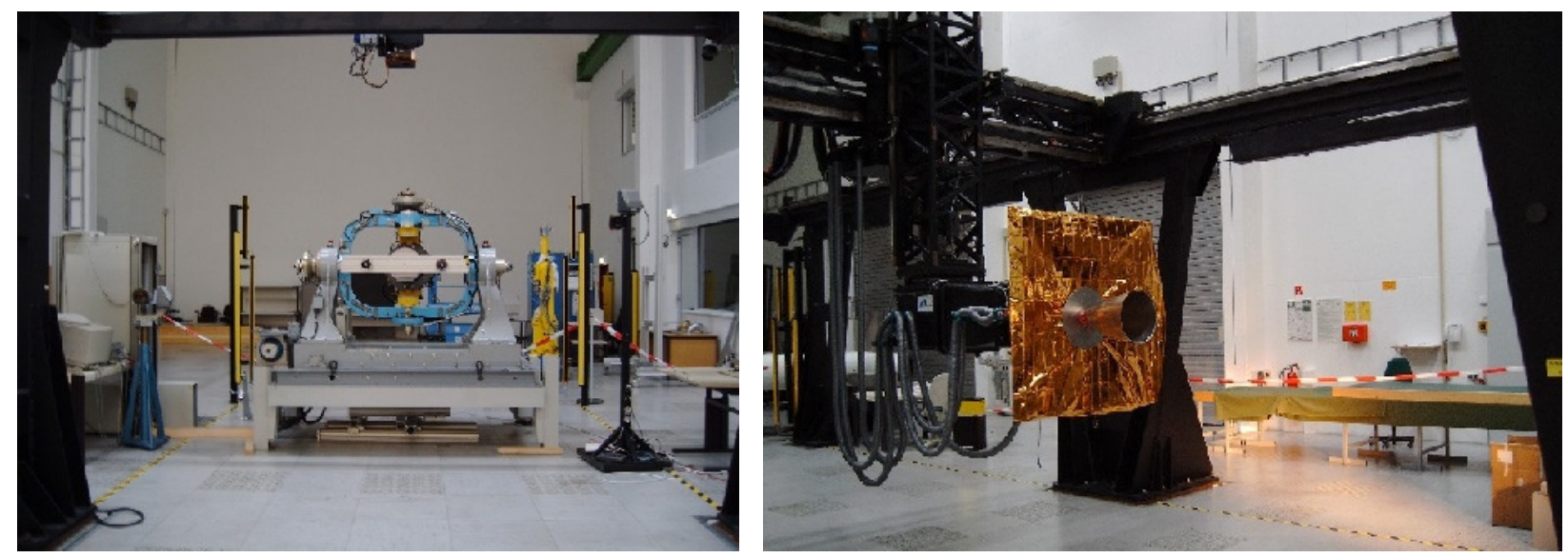

Figure 3. Old EPOS facility: the fixed part of the testbed (left) and the mobile part (right) 


\section{B. The New RvD Facilty - EPOS 2.0}

Future applications for satellite on-orbit servicing missions require the EPOS facility to be able to provide the following test and simulation capabilities

(A) the 6-DOF relative dynamic motion of two satellites in the final approaching phase from 25 meters to 0 meters.

(B) the 6-DOF contact dynamic behavior during the entire docking process including the initial impact, soft docking, and hard docking (final rigidization).

(C) the space-representative lighting and background conditions

Since the old EPOS facility apparently could not provide all of these capabilities, it was replaced completely by a new EPOS system. The design and construction work of the new facility began in 2008. The development work is a joint effort of two institutions of DLR at Oberpfaffenhofen. The first institution is the German Space Operations Center (GSOC), which provides the major resources for the project and is responsible for the overall design, construction, and operation of the facility. The second contributing institution is the DLR's Robotics and Mechatronics Institute, which provided expertise in space robotics technology and some HIL simulation experience.

The new EPOS facility is aimed at providing test and verification capabilities for complete RvD processes of onorbit servicing missions.. The facility comprises a hardware-in-the-loop simulator based on two industrial robots (of which one is mounted on a $25 \mathrm{~m}$ rail system) for physical real-time simulations of rendezvous and docking maneuvers. This test bed will allow simulation of the last critical phase (separation ranging from $25 \mathrm{~m}$ to $0 \mathrm{~m}$ ) of the approach process including the contact dynamics simulation of the docking process.

Moreover, its main advances are:

- It is a highly accurate test bed. The measurement and positioning performance will be increased by factor 10 compared to the former EPOS facility.

- Dynamical capabilities will allow for high commanding rates and the capability of force and torque measurements.

- The simulations of sunlight illumination conditions as well as the compensation of Earth-gravity force are both part of the assembly to generate an utmost realistic simulation of the real rendezvous and docking process.

- The utilization of standard industrial robotics $\mathrm{H} / \mathrm{W}$ allows a very high flexibility related to different application scenarios.

The new facility consists of the following components (for details, see ref. 17):

- A rail system mounted on the floor to move an industrial robot up to a distance of $25 \mathrm{~m}$,

- A KUKA KR240 robot (robot 1) mounted at the end of the rail system for simulating the 6 degree of freedom of the second spacecraft.

- A KUKA KR100HA robot (robot 2) mounted on the rail system for simulating the 6 degree of freedom of one spacecraft.

- A PC-based monitoring and control system to monitor and control the RvD simulation on the facility. It can be
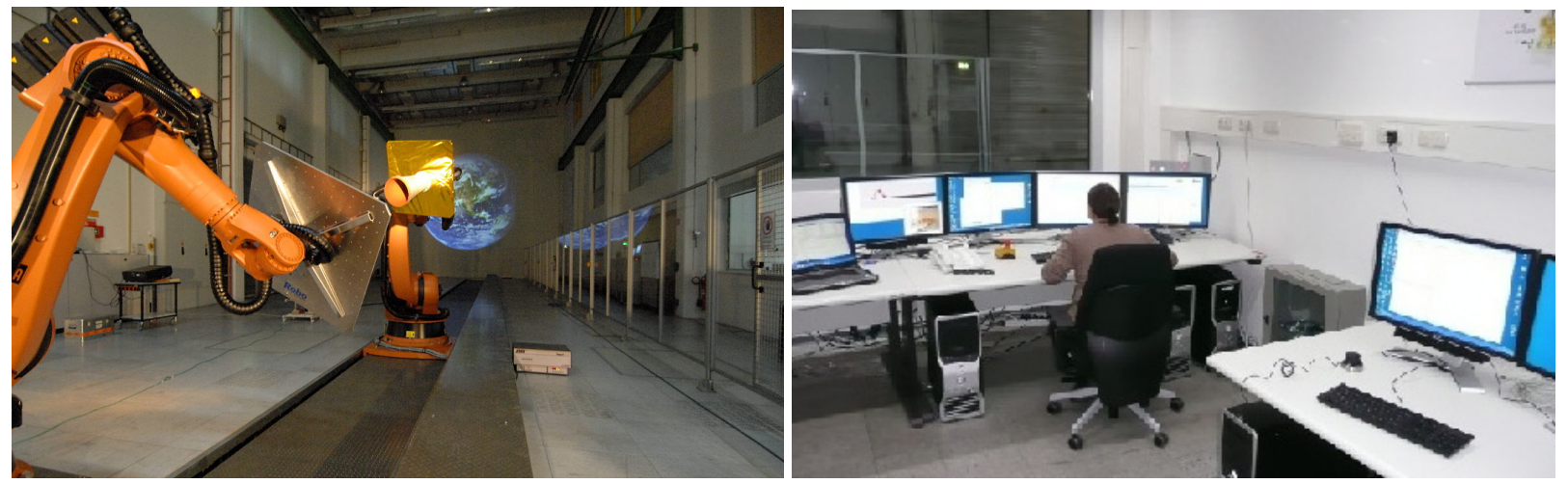

Figure 4 The new EPOS facility: robotics-based testbed (left) and operation station (right) 


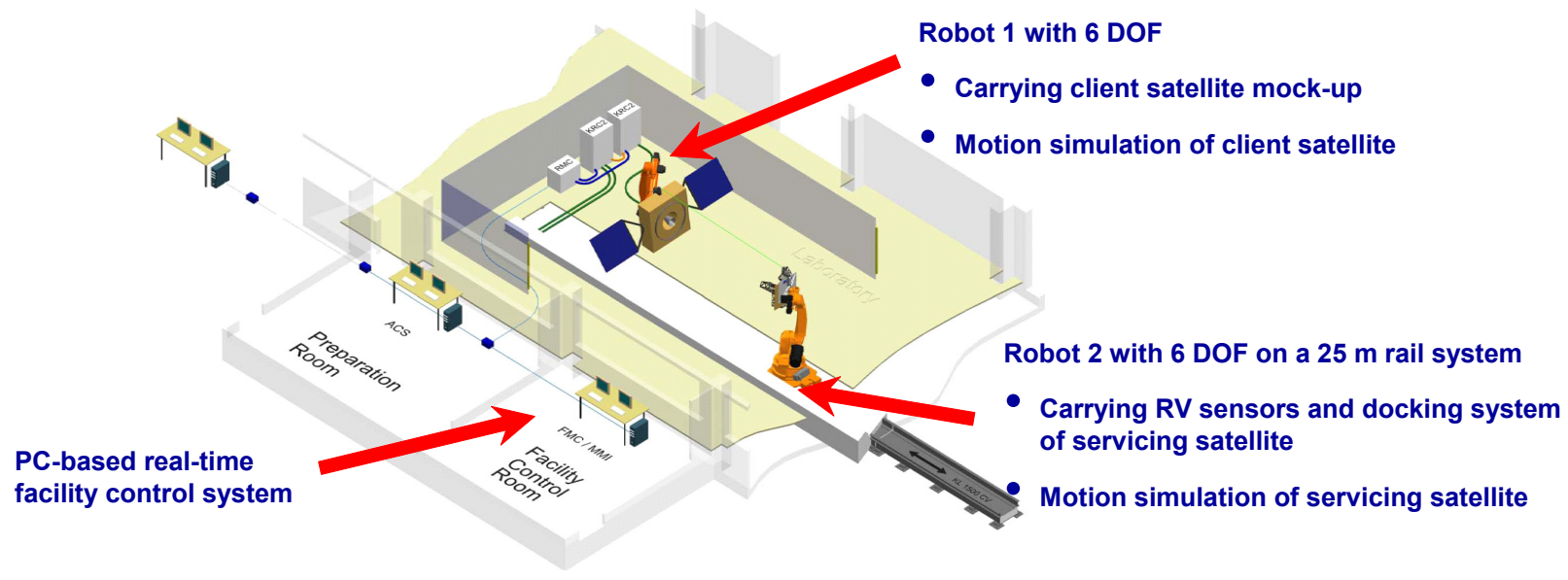

Figure 5 Components of the new testbed - EPOS 2.0

divided into three levels.

- The local robot control where all axes of the robots are separately controlled.

$\circ$ The facility monitoring and control system (FMC) where the entire facility is controlled in real time.

- The application control system where the actual RvD-simulation application is running

\section{Capabilities and Performances}

\section{Motion Ranges}

In order to simulate the rendezvous approach an additional translational degree of freedom is required, being realized by means of the highly accurate linear slide ( $25 \mathrm{~m}$ length). Each robotic arm in turn has 6 joints and thus 6 degrees of freedom. The EPOS motion ranges are being summarized in tab. 1:

\begin{tabular}{|l|c|c|c|c|}
\hline EPOS motion ranges & $\begin{array}{c}\text { Robot 1 } \\
\text { (ground fixed) }\end{array}$ & $\begin{array}{c}\text { Robot 2 } \\
\text { (on rail) }\end{array}$ & Rail & Complete \\
\hline degrees of freedom & 6 & 6 & 1 & 13 \\
\hline translational motion range & $\pm 2.2 \mathrm{~m}$ & $\pm 2.1 \mathrm{~m}+$ rail & $25 \mathrm{~m}$ & $\begin{array}{c}\text { Geometric workspace: } \\
25 \mathrm{~m} \mathrm{x} 4.2 \mathrm{~m} \times 4.2 \mathrm{~m}\end{array}$ \\
\hline rotational motion range & $\begin{array}{c}\text { average } 220^{\circ} \\
\text { per axis }\end{array}$ & $\begin{array}{c}\text { Average } 220^{\circ} \\
\text { per axis }\end{array}$ & -- & $\begin{array}{c}\text { roll: } \pm 600^{\circ} \\
\text { pitch: ca. } \pm 90^{\circ} \\
\text { yaw: ca. } \pm 90^{\circ}\end{array}$ \\
\hline
\end{tabular}

Table 1. FPOS motion ranges

Experimental test-setups have proven the roll-axis to be the most critical limitation of rotational motion. This is due to the fact that the simulation of a tumbling satellite's trajectory requires up to several hundred degrees of rolling.

Also the limitation of translational motion set by the length of the linear rail does impose a limit to the possible simulation scenarios, limiting the possible simulation setups with true-scale satellite models to $25 \mathrm{~m} \mathrm{RvD}$ maneuvers. This range can be extrapolated though by the use of downscaled mockups. 


\section{Accuracy}

The facility's positioning accuracy is required to be in the millimeter range, or even better in the submillimeter range. This is due to the fact that the simulation scenarios include missions with the target satellite's apogee-enginenozzle being used as a docking interface. The servicing satellite is being equipped with a docking-tool that has been precision engineered by the DLR to fit exactly into the target nozzle's inner throat, which has a diameter of only $16 \mathrm{~mm}$. This imposes the EPOS facility's positioning accuracy to be at least in the millimeter range.

The following table summarizes the EPOS positioning accuracy:

\begin{tabular}{|l|l|l|l|}
\hline \multicolumn{3}{|l|}{ linear positioning accuracy $\mathbf{( 3 D , 3 \sigma )}$} & \multicolumn{3}{l|}{ (atasheet specification } & measured \\
\hline & required & $\pm 0.12 \mathrm{~mm}$ (ISO 9283) & $0.3 \mathrm{~mm}$ \\
\hline Kuka KR 100 HA & $0.5 \mathrm{~mm}$ & $\pm 0.12 \mathrm{~mm}$ (ISO 9283) & $0.3 \mathrm{~mm}$ \\
\hline Kuka KR 240 & $0.5 \mathrm{~mm}$ &
\end{tabular}

\begin{tabular}{|l|l|l|l|}
\hline \multicolumn{4}{|l|}{ angular positioning accuracy $(\mathbf{3 D}, \mathbf{3 \sigma})$} \\
\hline & required & datasheet specification & measured \\
\hline Kuka KR 100 HA & $0.02^{\circ}$ & not specified & $0.2^{\circ}$ \\
\hline Kuka KR 240 & $0.02^{\circ}$ & not specifed & $0.2^{\circ}$ \\
\hline
\end{tabular}

\section{Table 2. EPOS accuracies}

The measurements have been carried out with a Leica laser tracker, allowing 3D precision measurements with micron-accuracy (absolute distance meter) and absolute angle detection with 0.5 " accuracy (ISO17123-3) with 0.07" resolution.

Table 2 indicates that the linear positioning accuracy is well within the required limits of $0.5 \mathrm{~mm}$. This is not really surprising as a standard industrial robot serves for exactly this purpose: Pick-and-place applications like in the automative industry require reliable repeat accuracy in the mass production. But when it comes to angular positioning accuracy, industrial robots come to struggle: They are not really designed for highly accurate angular accuracy whereas EPOS requires it. Nevertheless the measured angular accuracy of $0.2^{\circ}$ should still be sufficient for most test cases.

\section{Dynamic Performance}

EPOS' dynamic performance is highly important especially for docking contact dynamics. In order to carry out a stable docking contact dynamics simulation with EPOS, tests in the past have shown that a $4 \mathrm{~ms}$ command-frequency is the minimum requirement for reliable testing. Unfortunately the current industry-standard for industrial robots in the heavyload class $(\geq 100 \mathrm{~kg})$ is $12 \mathrm{~ms}$ only. Some suppliers have only recently introduced new controllers with a $1 \mathrm{~ms}$ command frequency, but only for small and lightweight robots up to $40 \mathrm{~kg}$ payload.

DLR was being provided with a new 4ms-prototype robot-controlller in order to meet the requirements for docking tests.

So far, the facility works well with the new prototype. As EPOS is a modular design allowing to exchange components and replace them seperately without the need to replace the system as a whole, the controllers can also be updated in the future when faster devices should be available in the future.

\section{Project Status of EPOS and Future Planning}

After dismantling the former EPOS facility in 2008 the design of the new facility was started. The new robotic hardware was installed in January 2009 and the entire facility monitoring and control system was finalized in October 2009. Now the next steps are under development::

- An Online-Measurement system to achieve higher simulation accuracy

- A sun simulator for utmost realistic simulation of illumination conditions

- Contact dynamic capability for including the docking simulation (see chapter V.) 


\section{Using EPOS for Rendezvous Navigation Developments}

At an early phase of this development there is no need of complete closed loop simulation of the entire rendezvous process. At this time the generation of realistic images or videos is enough. Therefore the facility is used in open loop means running predefined trajectories on the facility and generate the desired image sequences.

\section{A. Trajectory and Image Generation}

As a first part, the EPOS facility has been used to generate imagery of the satellite mockups for the design of the pose estimation algorithm described later in this paper. To this purpose, a motion command generator (MCG) has been developed. It is capable of performing linear motions. Using the MCG, an image sequence can be generated to test the pose estimator.

The EPOS MCG is a MATLAB based program consisting of functions, which allows the user to build up and simulate any kind of asynchronous trajectory commands. These functions can generate acceleration phases, a phase with constant velocity and deceleration phases. All calculated accelerations are based on a squared sinus function to avoid any step in the acceleration profile. Output of the functions are a list of robot positions and orientations with the sample time of the EPOS facility. With these functions it is very easy to build up complex command queues for simple user applications (see figure 6). A typical application is the generation of user-defined trajectories for rendezvous sensor tests. MATLAB was chosen as the host development program because of its mathematical and matrix solving capabilities. In addition Matlab provides an extended data visualisation and plotting capability for analysis of the generated trajectories.

For capturing images, a Prosilica Gigabit Ethernet Vision camera (GC-655) has been used (Figure 7). It is a monochrome sensor with very high sensitivity delivering up to 90 frames per second at VGA resolution. What is much more important here is, however, the synchronization capabilities. The camera has trigger inputs which can be used to trigger the acquisition. This
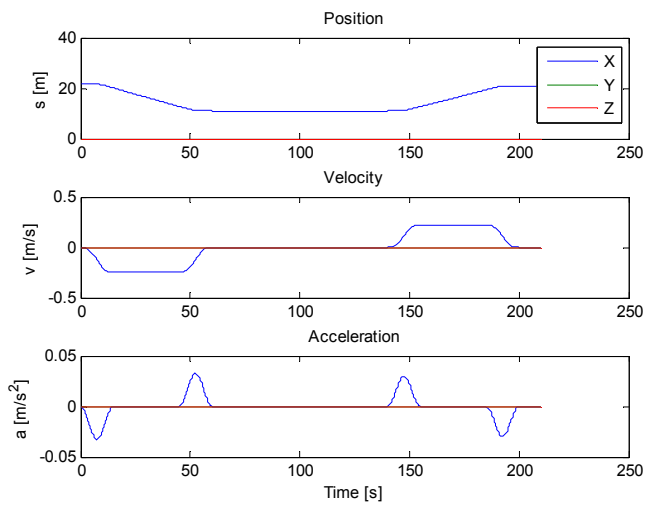

Figure 6 Visualisation of MCG Trajectory Sequence wit position, velocity and acceleration profike

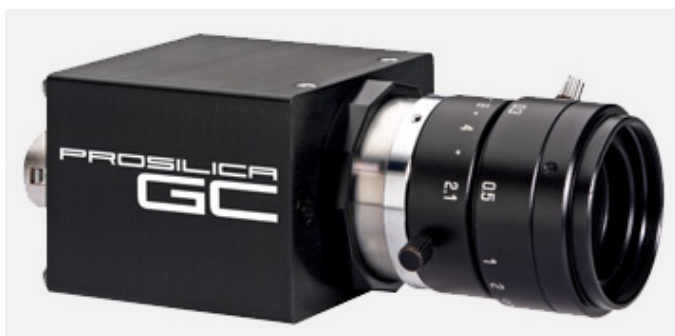

Figure 7 Prosilica Gigabit Ethernet Vision camera (GC-655)

way, it can be automatically synchronized to the facility. This synchronization is planned for the future and will be implemented soon.

To date, the camera is used in conjunction with a very simple image capture application which allows capturing images with a specific framerate set. Images are compressed using the lossless HUFFYUV codec and stored on-disk in an Audio-/Video Interleave (AVI) file for later processing.

The images obtained are processed offline using MATLAB. This allows automatic code generation in $\mathrm{C}$ for later implementation in the HIL scenario. Furthermore, it allows very fast design and optimization of the algorithm.

\section{B. EPOS Setup for a Geo-stationary Rendezvous Application}

Using a camera there is no need to simulate the entire rendezvous range in real size. For this type of sensor without a real distance measurement scenario scaling is an appropriate method: There are two options of scaling:

- $\quad$ Scaled size of model

A scaled model 1:10 extends the range by factor 10

- $\quad$ Scaled camera field of view (FOV)

A two times larger FOV extends the range by factor 2 
At EPOS both options are used. One satellite mockup has an scale of $1: 1$ and is showing only the side which is seeing at the close approach. The other satellite model is a scaled model 1:30 and is showing the entire geostationary satellite including solar panels and the large antennas. The models are shown in fig. 8 .

For the camera optics a typical mid range camera FOV (ca. 40 deg) is used. Apply this FOV to a far range camera with an FOV of $4 \mathrm{deg}$ an additional scaling by the factor 10 is possible.

A typical approach startegie to a Geo-stationary client satellite is shown in fig. 9. The relative
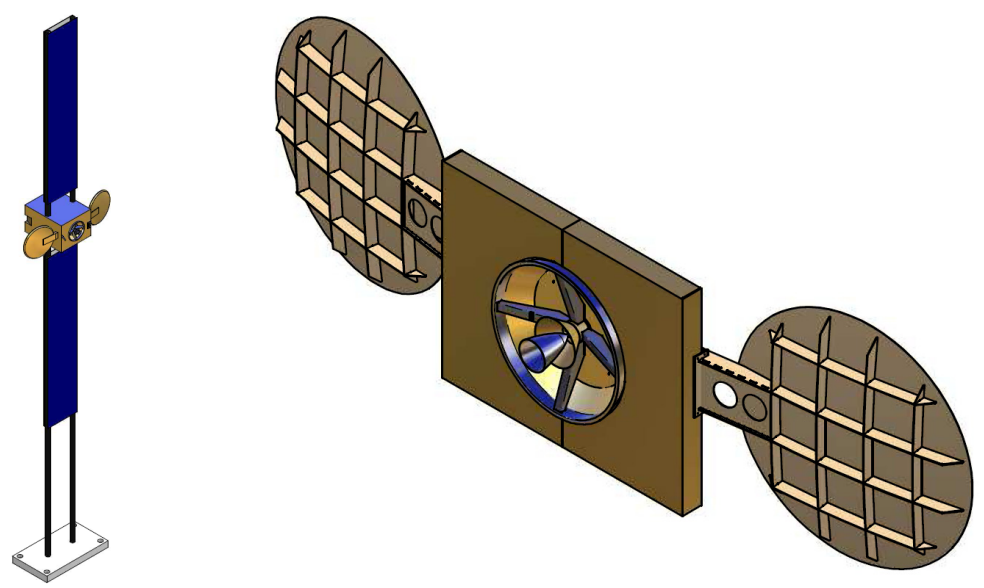

Figure 8 Used satellite mockups (left 1:30, right 1:1)

navigation starts at $2 \mathrm{~km}$ on V-Bar using a far range camera with small FOV (<4deg). At $100 \mathrm{~m}$ the GNC system switches from the far range camera to the mid range camera. At the same position the service satellites starts with a fly around ending at $50 \mathrm{~m}$ distance on R-Bar. From this point ist moves directly to the client.

The far range approach showing bad illumination conditions (see figure at $2 \mathrm{~km}$ ) due to the fact that the solar panels don't reflect the sun toward the cameras of the service satellite so the satellite body can only be seen in the image. The other image of the fare range camera is showing the difficulty of real sun simulation because the target satellite is not well illuminated instead the laboratory wall reflects the light.

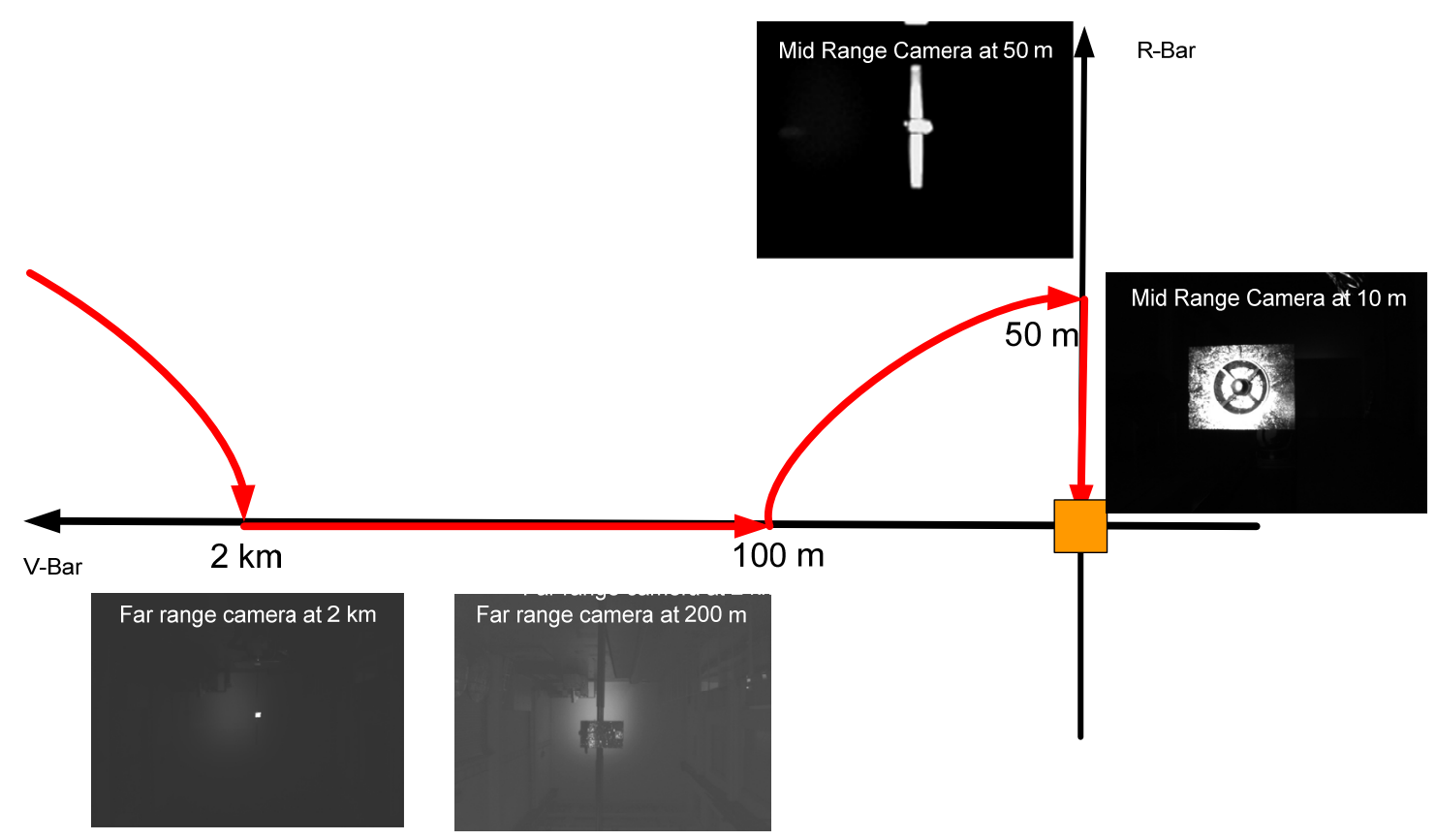

Figure 9. Rendezvous scenario and the generated images on EPOS 


\section{Image processing of generated images}

There are two different approaches being implemented serving two different distance ranges. First, there are methods for tracking the target in close range, providing full six-degrees of freedom information. Second, there are also methods for tracking the target at a large distance. In this case, however, only the direction to the target and its approximate distance can be estimated.

The close-range method (see fig. 10) is based on a hybrid edge tracker, which relies on classic edge tracking using the ADM detector ${ }^{14}$ in situations where this algorithm provides stable results. In cases of low contrast, where all classic edge tracking algorithms fail, a more recent method is used: texture segmentation. The choice of algorithm depends on the confidence indicator given by both algorithms. In practice, this provides a quite stable estimate of outer edges of the target object. Combined with knowledge about its size, sufficient information is

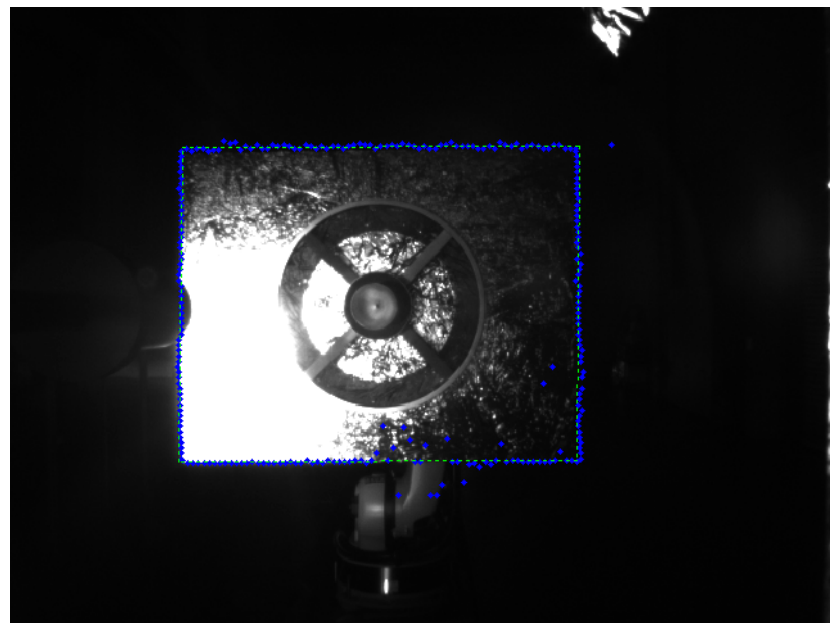

Figure 10. Edge tracking method for close range application

available to estimate the full 6-DOF of the target object. This method, however, needs a very accurate model and the object itself needs to be of a shape which provides a sufficient number of edges to detect. It will also work with cylindrical objects as long as there is some surface texture which allows to estimate the orientation. Details of this method can be found in another recent publication ${ }^{15}$.

The far-range method (see fig. 11) employs a list of different filters like edge filters, histogram filters, and so on. These filters can be applied in parallel. Their responses are weighted and combined. The resulting matrix provides a probability-of-presence map, which is then clustered using a cluster detection algorithm. After the properties of the clusters have been determined, a cluster selection algorithm reduces the data amount down to one single cluster. Finally, the central point of the cluster is determined, as well as its size. The size and intensity is a crucial part of the information needed for determining the approximate distance in the next step. After that, the direction and approximate distance to the target object is known. The gray parts shown in fig. 11 are planned to be implemented next. These parts will make the algorithm much more stable against light flashes, for example. Using the EPOS facility, these special situations can be encountered when moving the target. There is a 5 kilowatts theater lamp available as a basic sun simulation, where several reflections can be seen as soon as it is powered up. In the long run, software-generated imagery is planned to complement the images generated on EPOS. This way, the designed algorithms can achieve a maximum of robustness.

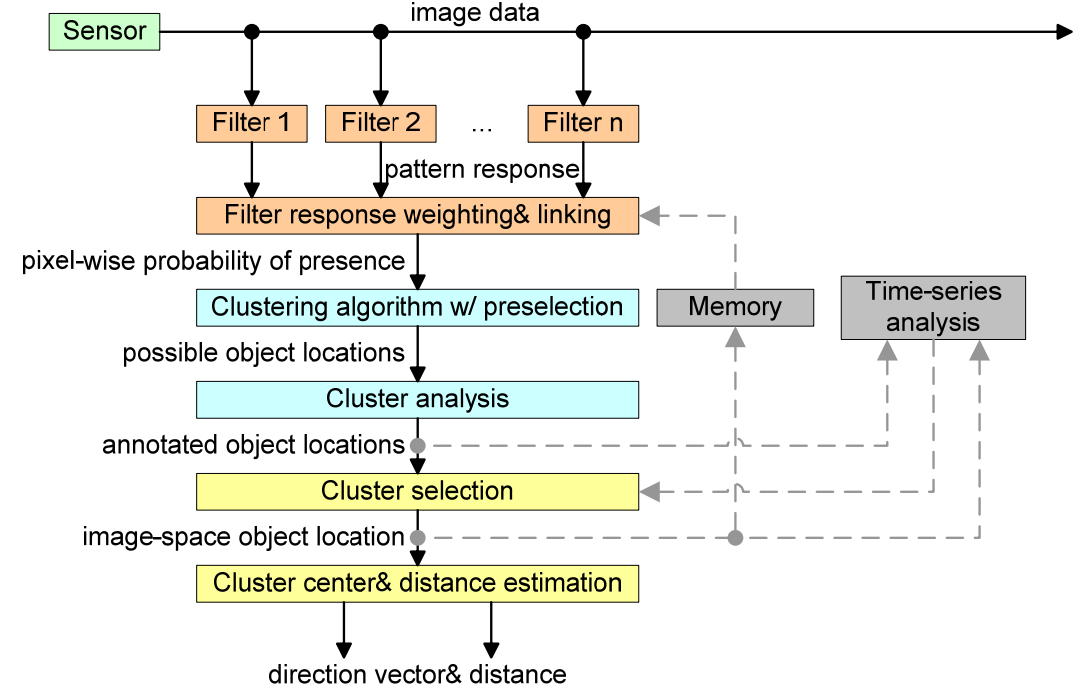

Figure 11. Far-range object position estimation flow diagram. The image is supplied by the sensor in the upper left. Several filters process the image and after a thorough cluster analysis, the direction and distance to the target is known. Gray parts indicate extensions which have not been completed yet. 


\section{Closed Loop Applications}

\section{A. General Scenario}

A typical set up of the EPOS facility for a DEOS RvD simulation scenario is shown in fig. 12.

For such "hardware in the loop" scenario the RvD sensors and the robotic manipulator arm are mounted on one robot and a typical satellite mockup of the client satellite is mounted on the other robot. The $\mathrm{RvD}$ sensors can measure the relative position and attitude of the client satellite and the onboard computer calculates on this basis the necessary thrusters or reaction wheel commands. These will feed in a real time simulator. This dynamic simulator computes for the next sample an update of the state vector (position / attitude of the spacecrafts) based on all relevant environmental and control forces and torques. Then the state vector for the new sample will be commanded to the facility.

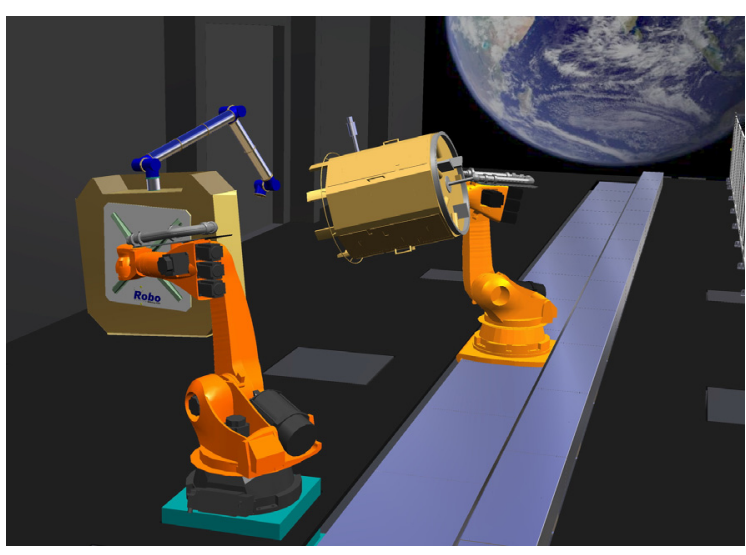

Figure 12. EPOS simulation set up for DEOS

Such a scenario can be used for a full RvD system test.

The system integration test concept for the OLEV scenario is shown in fig. 13. As shown, the scenario integrates the EPOS facility, the satellite simulator (OSTF HIL Simulator), the control center infrastructure like Operations Control System (OCS), Payload Control System (PCS, including Merger) and parts of the communication architecture (e.g. CORTEX).

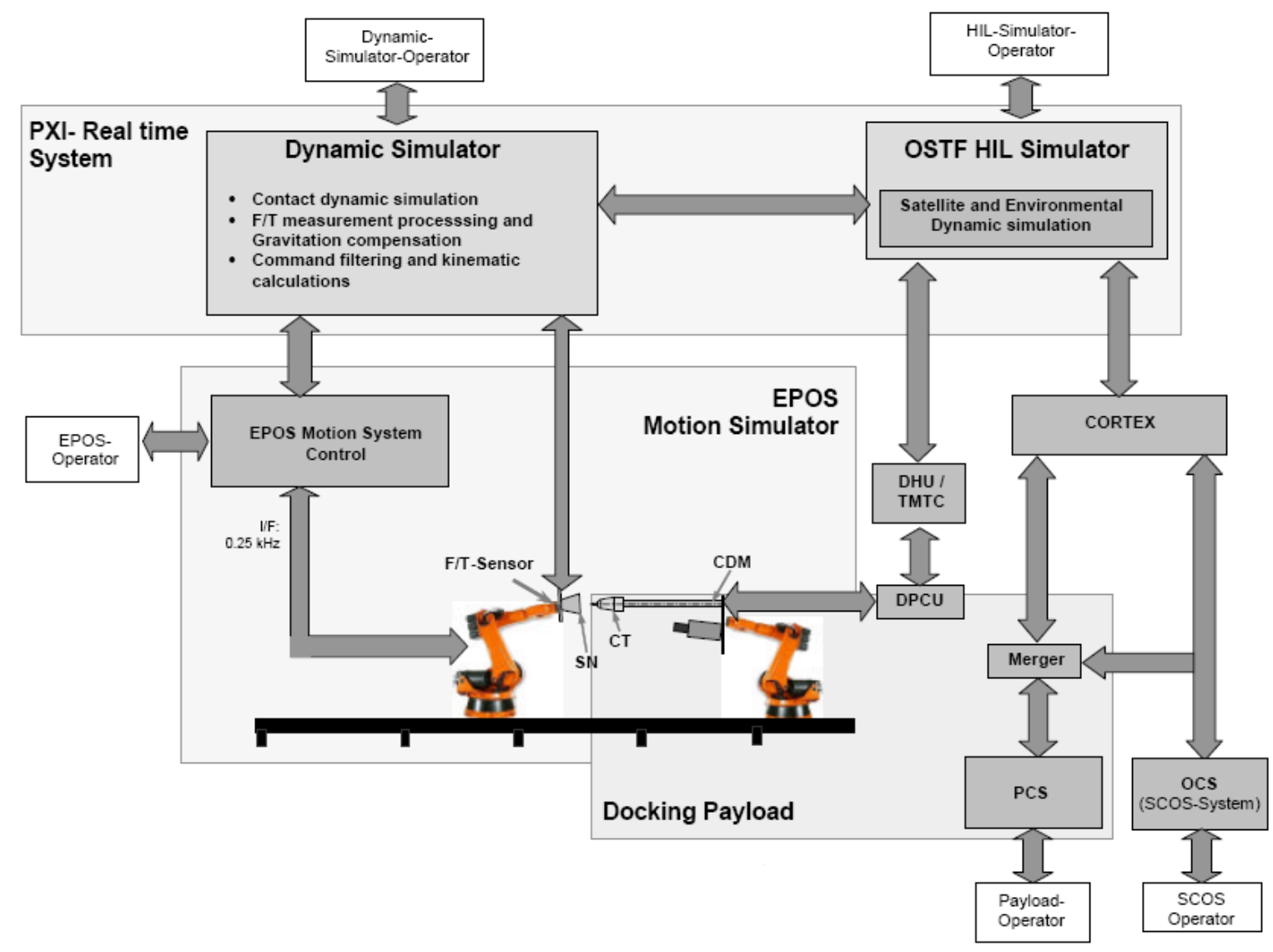

Figure 13. Possible EPOS HIL simulation scenario for OLEV 


\section{Contact Dynamic Simulation}

Physical contact in space is highly risky because the resulting contact forces can damage the satellites or destabilize its attitude control system. Therefore, any planned contact operations must be thoroughly evaluated and verified on the ground before such a mission can be launched. Due to the extreme difficulty of physically testing the system dynamics of large satellites in full 3D space and zero-gravity condition in lab environment, the technology of hardware-in-the-loop simulation (also called software-hardware hybrid simulation) is increasingly been applied for testing and verification of contact dynamics operations of space systems. NASA built a pilot-in-the-loop facility to investigate the berthing of Space Shuttle into the Space Station by the Shuttle Remote Manipulator System (also called Canadarm $)^{1}$. The berthing operations were performed using the real hardware driven by a computer simulation model of the robotic arm. A prediction-based feed-forward filter was used to make a ground-based Stewart-platform manipulator to generate contact forces and rebound velocities that match those expected during the same on-orbit operations ${ }^{2}$. DLR also did much study of the hardware-in-the-loop simulation technology and demonstrated that the criticalness of a fast data sampling rate for the stability of such a software-hardware hybrid simulation ${ }^{3}$. Canadian Space Agency developed a HIL contact dynamics simulator called SPDM Task Verification Facility (STVF) for studying and verifying the ISS robot SPDM performing contact tasks on the International Space Station $^{4}$. The greatest challenge of any robotics-based HIL contact dynamics simulation is that the facility robot or robots used to deliver the dynamic motion of the simulated space system must dynamically behave like the simulated space system during various delicate contact tasks ${ }^{5}$. In order to achieve this goal, many advanced robotics technologies have been tried in the above-mentioned existing HIL simulation facilities such as the torque-controlled joint servos, computed torque control with Cartesian linearization, impedance control, robust control, high bandwidth and precision force and motion censors, etc. However, not all of these advanced robotics techniques can be applied to every HIL system because their application requirements may not be met by the ground robot. This is indeed the main challenge for the EPOS system as described in this section.

\section{A. Control Architecture and Requirements}

The architecture of the high-level EPOS control system is illustrated in the diagram shown in fig 14. From the figure, one can see that the two active robots (KUKA industrial robots in this case) are controlled to strictly track the satellite simulation output. When a physical contact happens, the contact force and moment generated by the docking hardware will be fed back into the satellite simulator and, as a result, the simulated satellite motion output will be changed (with a time delay) by the contact forces. For such a HIL simulation system to have high simulation fidelity, it is required that the simulated docking behavior (which is measured by the resulting motion state and contact force-mention) must be the same as that from the real satellite docking operation. Based upon the common sense in engineering principle, one can easily understand that such a fundamental requirement can be achieved in the

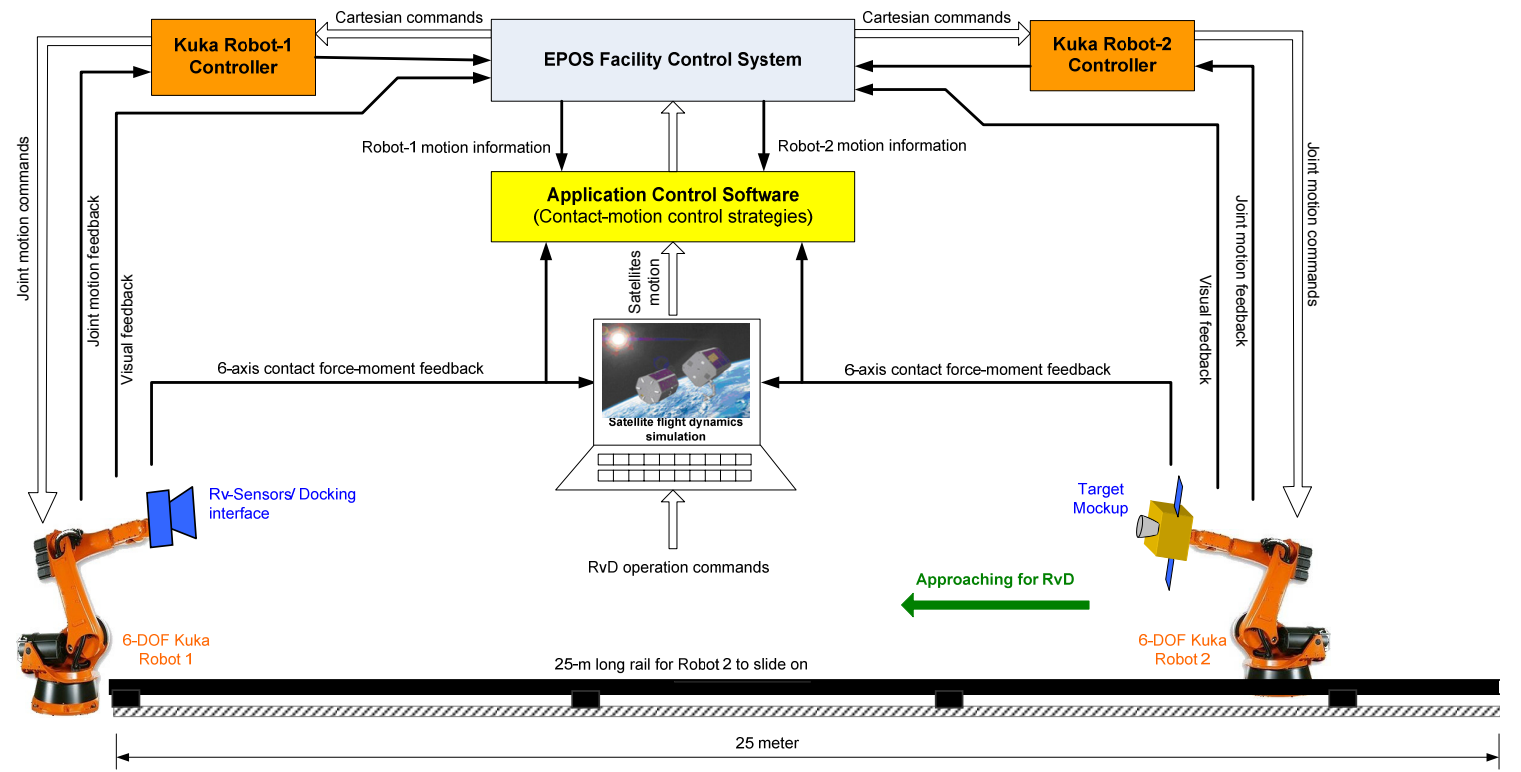

Figure 14. Control system of the EPOS facility 
following two conditions:

1) The active robots used to deliver the simulated satellite motion must be able to respond the HIL control command very fast.

2) When reacting to a physical contact during a docking operation, the active robot or robots (at its tip) must dynamically behave like the on-orbit satellites being simulated.

The first condition requires the active robots to have fast response to its control system and the second condition requires the robots to exhibit the same dynamics characteristics as the flying satellites to be simulated.

\section{B. Control Strategy}

Although the necessity of the first condition stated in Section V.A is not difficult to understand, it is not easy to meet for the EPOS robotic system because the two KUKA robots are industrial robots with massive bodies (thus large inertias). They are made for usual industrial applications, such as working in an automobile assembly line, and thus the robots do not have very fast responding speed. The known responding time of the robots is up to 8 command cycles and each command cycle takes 4 milliseconds ${ }^{6}$. In other words, the duration from the time when the EPOS control system issues a control command to the time when the robot physically reacts to the command can be up to 8 command cycles or $32 \mathrm{~ms}$. This is a large time delay for controlling a robot to perform contact motions. Moreover, the maximum sampling rate of the robots is the same as their commanding rate, $250 \mathrm{~Hz}$. This rate has been quite high for a usual industrial robot but it is not considered high for a robot to be used to perform HIL contact dynamics simulations. Since the KUKA robots must be used as is in the EPOS HIL system, special process control system must be developed to resolve this time delay problem. The Robotics and Mechatronics Institute of DLR is currently developing technology to practically solve this problem. Their intended approach is based on the principle of actively balancing the energy inputting to and outputting from the robotic system. In other words, the method is trying to achieve a passive behavior of the industrial robots in the HIL simulation process, so that the risk of simulation instability due to the large time delay will not happen. An introduction of the said technology has been reported in ref. 7.

Obviously, the second condition described in Section V.A cannot be met by the industrial robots either because the robots are basically positioning machines with high stiffness. Therefore, when the robot tip is in a physical contact or being pushed by an external object (e.g., the docking hardware), it will not comply as a free-floating satellite would. The robot may even encounter instability in a stiff contact case. Note that whether a contact operation to be simulated is stiff or not depends on the satellites and their docking interfaces and it cannot be chosen by the EPOS system. Therefore, another control loop outside the industrial robot needs to be implemented to solve this problem (since no inner control loops of the industrial robots can be modified). An end-effort contact force control method cannot be implemented either because the reference contact force profile for a proper docking operation can never be known. An ideal approach would be to apply an impedance control strategy such as the one described in ref 9. The application of the admittance control technology in the Canadian HIL simulator STVF has showed robust performance against the variations of some parameters of the its robotics system as well as possible external disturbances ${ }^{10}$. However, an impedance control requires torque control capability at the joint level. This is not available with the KUKA robots. All we have from the robots is an end-effector position or rate control capability. Similarly, many other advanced and proven robot control strategies, such as the computed torque control $^{11}$, passivity-based control ${ }^{12}$, etc. cannot be implemented in EPOS either because we either do not have a joint torque control capability or do not have an inverse dynamics model of the robots. Instead, an end-effector admittance control strategy is proposed to deal with the problem because such a control method does not need a joint torque control capability or a dynamics mode of the robot to implement.

As seen in the block diagram shown in fig. 15, the admittance control will adjust the commanded end-effector trajectory (generated by the satellite simulator) such that the robot will exhibit similar impedance as the docking satellites during a docking operation. The input to the admittance control will be the contact force and moment which are measured from the physical contact between the two parts of the docking hardware. The admittance control law is expressed at high level as follows:

$$
\Delta X=\left(\mathbf{M} s^{2}+\mathbf{C} s+\mathbf{K}\right)^{-1} F
$$

where $\mathbf{M} \in R^{6 \times 6}$ is the inertia matrix of the impedance model; $\mathbf{C} \in R^{6 \times 6}$ is the damping matrix of the impedance

model; $\mathbf{K} \in R^{6 \times 6}$ is the stiff matrix of the impedance model; $\Delta X \in R^{6}$ and $F \in R^{6}$ are the Laplas transforms of the time-domain relative tip position and orientation $\Delta \mathbf{x}(t)$ and the contact force and moment vector $\mathbf{f}(t)$. It should be emphasized that the solution of $\Delta \mathbf{x}(t)$ depends not only on the inverse of $\left(\mathbf{M} s^{2}+\mathbf{C s}+\mathbf{K}\right)$ but also on the 


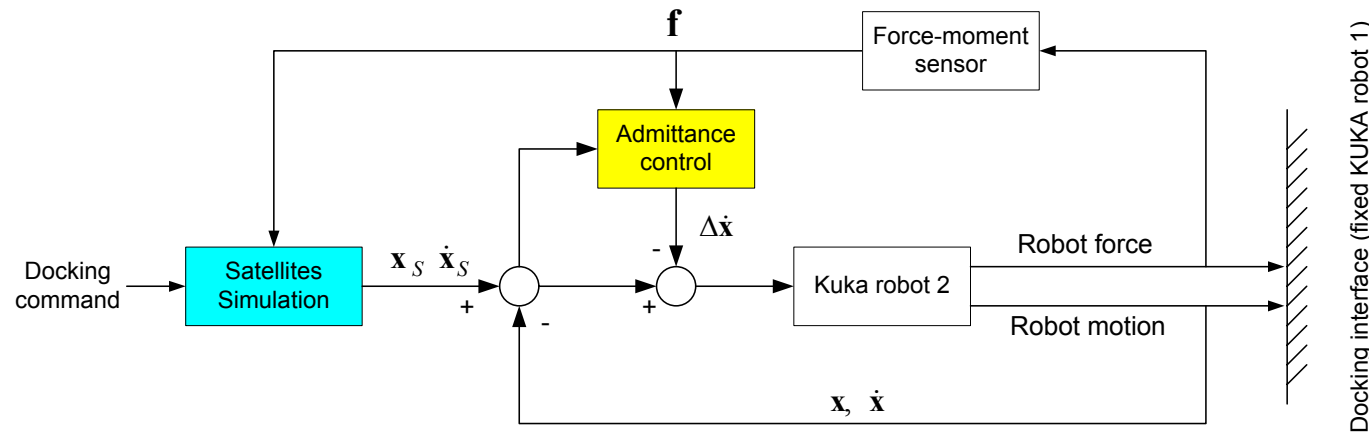

Figure 15. Admittance control strategy for EPOS robot

characteristics of the contact force $\mathbf{f}(t)$ as a function. To facilitate the implementation work, a modified admittance control scheme is proposed as follows:

$$
\Delta \dot{\mathbf{x}}=\mathbf{M}^{-1} \int[\mathbf{f}-\mathbf{C} \Delta \dot{\mathbf{x}}-\mathbf{K} \Delta \mathbf{x}] d t
$$

where only the inverse of the inertia matrix of the impedance model is required. Sine the inertia matrix is always assumed positive definite, its inverse is easy and accurate. The corresponding block diagram of the control method is illustrated in fig. 15. Note that this modified control method performs control to the tip velocity only. This is acceptable in practice as some other researchers suggested ${ }^{13}$.

It should be emphasized that the impedance model used in the admittance control algorithm needs to be carefully selected based on the dynamics properties of the involved satellites. This should not be very difficult if the dynamics properties of the two involved satellites are known.

\section{Simulation Example}

Before the implementation of the proposed HIL contact dynamics simulation method in the real EPOS facility, a simulation based study of the method and some other ideas have been conducted. In the study, a reduced EPOS HIL simulation system was modelled and implemented on Simulink. In the simulation model, one of the two robots is assumed to have 3 joints and the other one is fixed, as shown in fig 16, a similar setup as the real EPOS robotic system. The satellite system in the simulation study is a planar case as shown in fig. 17. Both satellites can have a full planar motion and each has compliance between its main body and the docking interface hardware. The docking interface is just a simple stick probe contacting cone case but its dimensions are the

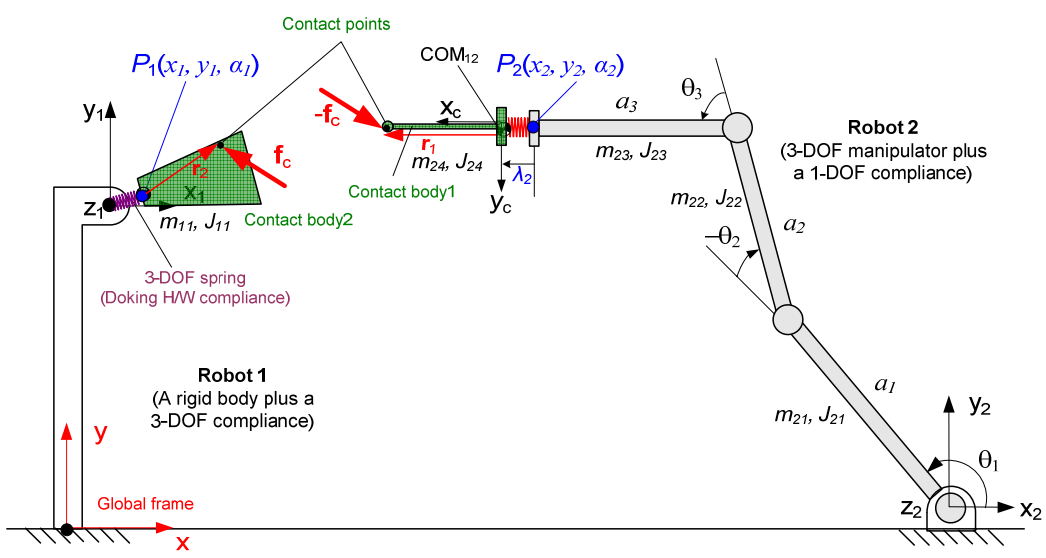

Figure 16. An example of EPOS HIL robotics system for studying the control concepts and options
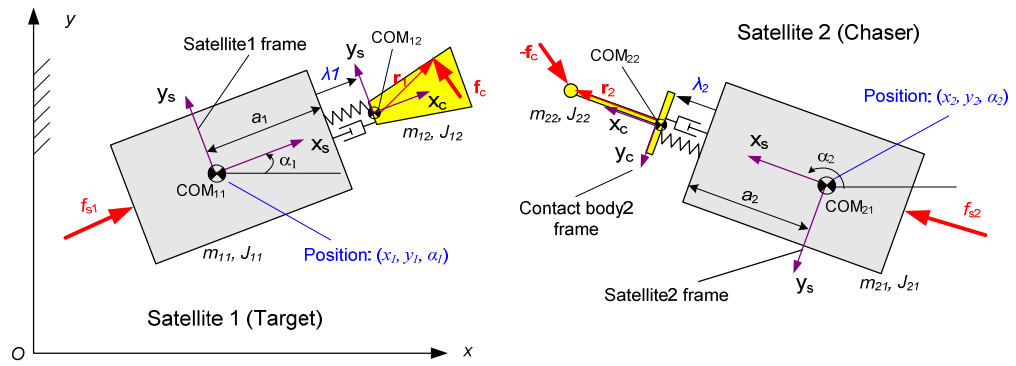

Figure 17. The satellite docking case to be simulated by the example EPOS HIL simulation system same as the benchmark docking interface hardware currently implemented in the real EPOS facility.

In the assumptions the masses of the two satellites are assumed to be $1500 \mathrm{~kg}$ and $750 \mathrm{~kg}$ respectively. The kinematics and dynamics parameters of the robot system are also set to be as close as possible to the real values of 
the EPOS robots. Since this is only a simulation study, the physical contact of docking is modelled by a contact dynamics model. The contact stiffness and damping parameters are set to $100000 \mathrm{~N} / \mathrm{m}$ and $2000 \mathrm{Ns} / \mathrm{m}$, respectively.

In the example, Satellite 2 is assumed to passively approach Satellite1 at an initial relative velocity of $5 \mathrm{~cm} / \mathrm{s}$ in the $-\mathrm{x}$ direction. The two parts of the docking interfaces have an initial lateral misalignment of $0.15 \mathrm{~m}$ in the $\mathrm{y}$ direction. No angular initial misalignment is assumed. In the simulation model, the relative tip motion between the two satellites is defined as the relative position, orientation, linear velocity, and angular velocity of the reference point $\mathrm{COM}_{22}$ of Satellite2 with respect to that of the reference point $\mathrm{COM}_{12}$ of Satellite1 (see fig. 17 for illustration). The relative tip motion between the two EPOS robots is defined as the relative position, orientation, linear velocity, and angular velocity of the reference point P2 of Robot 2 with respect to that of the reference P1 of Robot1 (see fig. 16 for illustration). The relative tip motion between the two satellites is the reference motion for the EPOS robotics system to track during the HIL simulation. Since EPOS Robot1 is fixed during simulation, EPOS Robot2 has to track the relative tip motion of the two satellites as opposed to just the tip motion of Satellite2.

When we set up a docking simulation case, we have to first determine the initial conditions. From application point of view, it is logical to first define the initial conditions of the two satellites and then calculate the corresponding initial conditions of the two EPOS robots because the EPOS facility is used to simulate satellites. This will have a problem because EPOS is a hardware system and it is nontrivial to set up any given initial tip position and velocity of a real robot system because the robot has to move from rest as opposed to from an arbitrarily given velocity. For this reason, in our simulation testing, we just make Robot 2 match the initial relative position of the two satellites, leaving the initial velocity as zero. The robot's control system will push the robot to track the satellite relative velocity soon after the robot starts to move. fig. 18 shows how quick the EPOS Robot2 can track the satellite relative velocity from zero initial velocity.

Shown in fig. 19 are the simulated contact force-moment

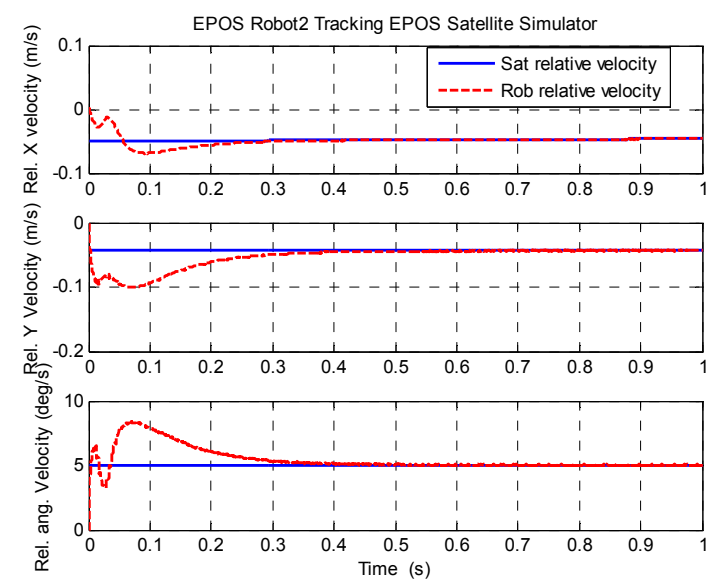

Figure 18. EPOS robot quickly tracks the nonzero satellite velocity after it starts to move from rest data also from an independent satellite simulation and those from the EPOS HIL Simulation. Since the independent satellite simulation is completely independent from the EPOS system, this comparison tells how accurately the EPOS HIL Simulation System has simulated the satellite docking case.
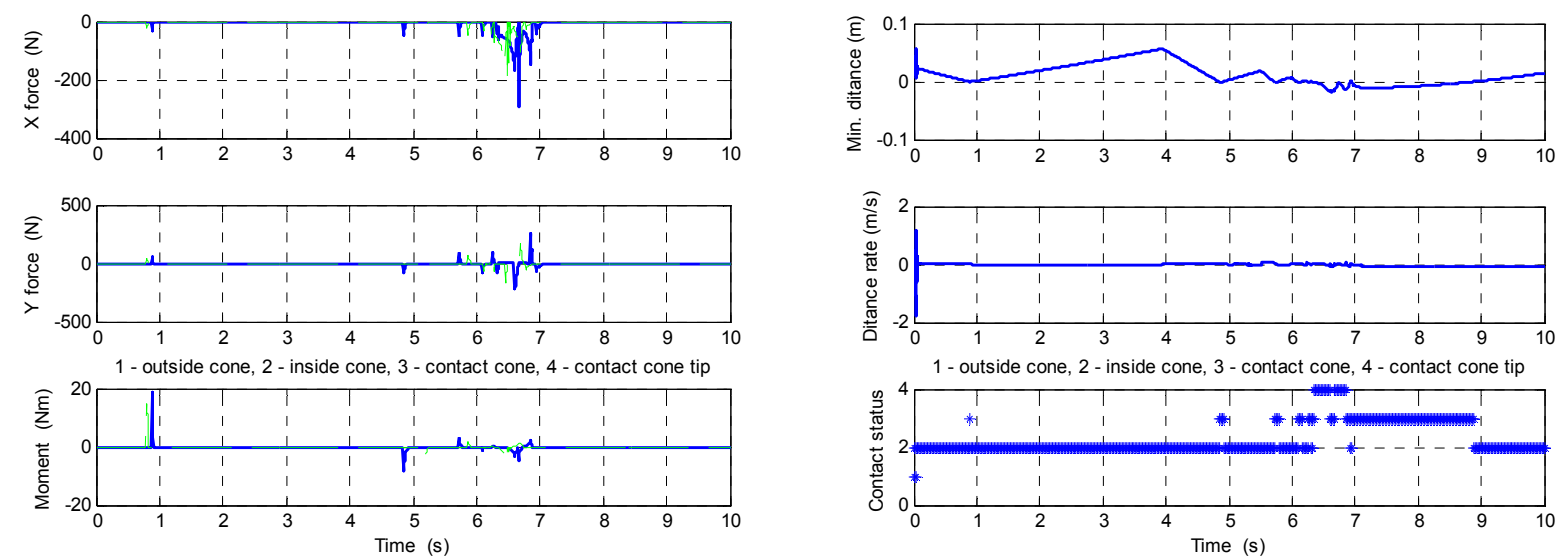

Figure 19 Comparison of the EPOS HIL simulation and the independent satellite simulation (left) and Contact status information (right) 


\section{Conclusions}

This paper gave an insight into the challenges concerning RvD processes for on-orbit servicing missions. Based on these, the new EPOS facility at DLR GSOC was presented in detail. It is designed for the simulation of RvD processes such as the ones shown in the beginning. In addition, an image generation process performed on the EPOS facility was described and used for the development of a first prototype of an image processing algorithm.

In the second part, the article summarized the current development stage of implementing the contact dynamic simulation capability on the facility.The entire control process is summarized and a first simulation example is given.

\section{Acknowledgments}

The authors want to thank Mr. Landzettel and Mr. Krenn from the DLR Institute of Robotic and Mechatronic for the fruitful cooperation on contact dynamic developments on EPOS.

Finally, we want to thank the Robo-Technology GmbH (Mr. Bosse) for supporting and building the new EPOS facility.

\section{References}

${ }^{1}$ S. Veerasarmy and J. Hubbard, "Real-time berthing for space station and space shuttle", Simulation, Vol.57, No.1, pp.31-38, 1991.

${ }^{2}$ S. Ananthakrishnan, R. Teders, and K. Alder, "Role of estimation in real-time contact dynamics enhancement of space station engineering facility”, IEEE Robotics and Auto. Mag., pp.20-28, 1996

${ }^{3}$ Rainer Krenn and Bernd Schaefer, "On HIL simulation dynamics by force/torque sensor feedback", Contact Dynamics Workshop, Montreal, Canada, June 22, 1999.

${ }^{4}$ M. Doyon, J.-C. Piedboeuf, F. Aghili, Y. Gonthier and E. Martin, "The SPDM Task Verification Facility: On the Dynamic Emulation in One-g Environment using Hardware-in-the-Loop Simulation", Proc. of 7th Inter. Symp. on Artificial Intelligence, Robotics and Automation in Space: i-SAIRAS 2003, NARA, Japan, May 19-23, 2003.

${ }^{5}$ O. Ma, J. Wang, S. Misra, and M. Liu, "On the validation of SPDM task verification facility", Journal of Robotic Systems, Vol.21, No.5, 2004, pp. 219-235.

${ }^{6}$ EPOS Facility Manual, Robo-Technology

${ }^{7}$ Rainer Krenn and Bernd Schaefer, "On HIL simulation dynamics by force/torque sensor feedback", Contact Dynamics Workshop, Montreal, Canada, June 22, 1999.

${ }^{8}$ B. Schaefer, R. Krenn, M. Loesch, and W. Rulka, "Space robotics dynamics simulation performance in real-time environments", Adances in Computational Multibody Dynamics, Lisbon, Portugal, Sept.20-23, 1999.

${ }^{9}$ N. Hogan and S.P. Buerger, "Impedance and Interaction Control", Chapter 19 of ????.

${ }^{10}$ AGHILI, E, DUPUIS, E., PIEDBaeUF, J.-C., and DE CARUFEL, J.:PERRY, M. Hardware-in-the-loop simulations of robots performing contact tasks'. Proceedings of fifth intemational symposium on Artificial intelligence, robotics and automation in space, 1999, Noordwijk, The Netherlands, ESA,.Publication Division, pp. 583-588.

${ }^{11}$ R. H. Middleton and G. C. Goodwin, "Adaptive computed torque control for rigid link manipulations", Systems \& Control Letters, Vol. 10, Issue 1, January 1988, pp.9-16.R.

${ }^{12}$ Ortega, A.J. van der Schaft, I. Mareels, and B. Maschke, "Putting energy back in control", IEEE Control Systems Magazine, February 2001.

${ }^{13}$ K. Yoshida, H. Nakanishi and H. Ueno, "Dynamics, Control and Impedance Matching for Robotic Capture of a Noncooperative Satellite," Advanced Robotics, vol.18, no.2, pp. 175-198, 2004.

${ }^{14}$ Alzahrani, F.M., and Chen, T.: “A real-time edge detector: algorithm and VLSI architecture. Real-Time Imaging”, 1997, 3, (5), pp. 363-378.

${ }^{15}$ T. Tzschichholz and T. Boge. GNC Systems Development In Conjunction With A RvD Hardware-In-The-Loop Simulator. In: International Conference on Astrodynamics Tools and Techniques, Madrid, Spain, May 2010.

${ }^{16}$ Mulder, T.A., "Orbital Express Autonomous Rendezvous and Capture flight operations", Part 1 of 2 and Part 2 of 2 , AIAA/AAS Astrodynamics Specialist Conference and Exhibit, 18 - 21 August 2008, Honolulu, Hawaii

${ }^{17}$ Boge, T., et al.: "Hardware in the Loop Simulator von Rendezvous und Docking Manövern”, German Aerospace Congress of DGLR, Aachen, Germany, 8-10 September, 2009

${ }^{18}$ Rupp, T., Boge, T., Kiehling, R., Sellmaier, F.: "Flight Dynamics Challenges of the German On-Orbit Servicing Mission DEOS ”, 21st International Symposium on Space Flight Dynamics, Toulouse, France, September/October, 2009

${ }^{19}$ Miravet, C., Pascual, L., Krouch, E., Del Cura, J.M., "An Image-based sensor system for autonomous Rendezvous with uncooperative satellites”, 7th International ESA Conference on Guidance, Navigation \& Control Systems, 2-5 June, 2008 